\title{
Improving Breast Cancer Education: The Case of an Evolving Multidisciplinary Module for Undergraduate Medical Students (Lausanne Medical School, 1993-2008)
}

\author{
Maryse Fiche • Domenico Lepori • Daniel Guntern • \\ Patrick Jucker-Kupper • Wendy Jeanneret • \\ Khalil Zaman • Sara Vadot • Jean-François Delaloye
}

Published online: 16 January 2010

(C) Springer 2010

\begin{abstract}
Breast cancer is a public health issue in numerous countries. Multidisciplinary collaboration is required for patient care, research, and also education of future physicians. This paper uses Kern's framework for curriculum design to demonstrate how a breast diseases module for undergraduate medical students created in 1993 evolved over 15 years. The main outcomes of program refinements
\end{abstract}

\footnotetext{
M. Fiche

Pathology, Imaging Radiology, Oncology, Radio-Oncology, and Gynecology Departments, CHUV,

Lausanne, Switzerland

D. Lepori · D. Guntern

Imaging Radiology Department, CHUV,

Lausanne, Switzerland

M. Fiche $\cdot$ P. Jucker-Kupper $\cdot$ S. Vadot

Education Unit, Faculty of Biology and Medicine,

University of Lausanne,

Lausanne, Switzerland

W. Jeanneret

Radio-Oncology Department, CHUV,

Lausanne, Switzerland

K. Zaman

Oncology Department, CHUV,

Lausanne, Switzerland

J.-F. Delaloye

Gynecology Department, CHUV,

Lausanne, Switzerland

M. Fiche $(\square)$

Unité Pédagogique Faculté de Biologie et Médecine Bugnon 21,

CH-1011 Lausanne, Switzerland

e-mail: maryse.fiche@chuv.ch
}

were better integrated course content, the development of electronic course documents, and implementation of computer-aided small group learning. A main future challenge is to further develop efficient instructional strategies in line with well-defined learning needs for undergraduate students.

Keywords Kern's framework for curriculum . Breast cancer - Undergraduate medical education . Multidisciplinary (medical) education · Curriculum design

\section{Introduction}

The high incidence of breast cancer in many countries, as well as ongoing evolutions in diagnostic and therapeutic strategies, result in a need to include this topic in undergraduate medical training.

The different specialists who collaborate in patient care, postgraduate education, and research may also be required to teach undergraduate students together. Cancer education must benefit from the integration of content and multidisciplinary cooperation, which have been shown to offer specific opportunities for effective learning and instruction in medicine $[1,2]$.

In this paper, we describe how a breast diseases integrated module for undergraduate medical students was initially created in 1993 at the Lausanne University Faculty of Medicine, as an answer to an emerging educational need, and then taught over 15 years by a changing multidisciplinary team. We want to demonstrate how sustained multidisciplinary cooperation combined with responses to progress in computer technology and educational knowledge resulted in a refined program in 
line with evolving requirements for training twenty-first century physicians.

\section{Method}

We analyzed the breast diseases module using Kern's framework for curriculum design. This framework emphasizes consistency between educational needs, learning objectives, instructional and assessment methods, and program evaluation [3]. We retrospectively describe how these elements were addressed at the time of initial implementation of the program in 1993 and how they evolved over 15 years, and finally we outline the perspectives for future development.

\section{Results}

1993: Creation of an Integrated Breast Diseases Course

\section{Learning Needs, Learning Objectives, and Course Content}

The breast diseases integrated course was introduced in the 4 th year of undergraduate studies (equivalent of 1st clinical year) at the Lausanne Faculty of Medicine in 1993. To our knowledge, no learning objectives were explicitly defined at that time. The course content and the invited faculty rather appeared as a balanced representation of several clinical specialties involved in breast cancer patients' care, with a radiologist as a coordinator. On the whole, eight faculty members from different departments (radiology, gynecology, oncology, surgery, psychiatry, and endocrinology) participated in instruction. Normal breast was followed by benign diseases, then by early and advanced breast cancer. Clinical examination, imaging techniques, surgery, and oncology were presented in turn within each section.

\section{Instruction Format, Students Assessment}

The integrated lecture-based course was organized into twelve 45-minutes-long lectures given within 1 week. Two or more teachers shared each session. The knowledge acquired by the students during this module in 4 th year was only assessed at the end of the 6th year, due to the federal regulations for examinations in Switzerland.

\section{Program Evaluation}

The course was evaluated once, in 2001, using a student paper survey. Unfortunately, the results could not be found for the present report (as a consequence of a loss of data in a computer crash). According to a former course director, students' satisfaction was overall high.

\section{3-2007: Evolving the Program}

The course was taught over 15 years by a changing group of faculty members, as some arrived or left the institution. Most of the changes reported hereunder occurred between 2000 and 2007-2008.

Learning Needs, Learning Objectives, and Course Content

While learning needs were never systematically assessed, faculty empirically and progressively adapted the course content to issues they perceived as clinically relevant. In 1998, pathology and plastic surgery were included.

The resulting course content was characterized in 2007, as compared with 2002 , by (1) less teaching time dedicated to benign breast diseases and more to breast cancer; (2) a refined structure of the content around an algorithmic approach of four clinical settings, e.g., patient with: a breast nodule; early invasive breast cancer; ductal carcinoma in situ; locally advanced breast cancer; and (3) more teaching time devoted to the psychological aspects of the disease and the patient-doctor relationship. In 2007-2008, a list of learning objectives was communicated to the students, in an attempt to formalize instruction methodology.

\section{Instruction Format and Course Documents}

Lectures, the only instructional method in continual use for this course during 14 years, were still predominant in 2007. All the didactic presentations progressively adopted an electronic format. They came to include many illustrations of the various procedures (e.g., imaging and surgical) involved in breast diseases diagnostic and treatment. After a long period of juxtaposed rather than truly integrated lectures, cooperation between specialist teachers resulted, from 2004, in common integrated course documents.

In 2007, a 2-hour interactive small group session was implemented, at Micropolis, a classroom developed at our medical school for small-group computer-aided learning [4]. Each group of four to seven students worked around a table equipped with a two-headed microscope and a computer, on two different clinical cases presented via the computer. Students were invited to choose a role of a specialist per group and to progress along the cases through the pre-designed electronic file. Their task included making mock basic decisions about imaging, surgery, and oncology. At mid-session, the "specialists" from all small groups met together and with the corresponding "senior specialist" (e.g., all radiologists with the senior radiologist, surgeons 
with senior surgeon) to further discuss their specific problems with the cases. Afterwards, they went back to their table to share with other students the knowledge they had gained, and agree a final electronic document per group. A final quiz, mostly image-based, closed the session by revisiting key concepts and facts. In parallel to this main learning activity, students attended in turn two separate workshops. In these, they could manipulate the devices used for trans-cutaneous biopsy with the radiologist and examine a dozen mastectomy specimen (inside plastic pockets) with various lesions with the pathologist. Faculty members from five different disciplines participated in the session, answering questions and clarifying content presented during the lectures. Finally, students could save the electronic file for later review.

\section{Learning Resources}

From around year 2000, Pathology course documents became available for students on the Pathology Institute's website. In 2007, most of the didactic electronic files presented in lectures were made available for students via the university intranet before the lectures. In 2007 too, a recently designed web tool (primarily designed for computer-aided auto-learning) replaced the video demonstration of the clinical examination procedure filmed in the 1980s [5].

\section{Program Evaluation}

The program was evaluated in academic years 2006-2007 (partially) and 2007-2008 (whole module) using students' paper surveys. Evaluation results were globally positive (Fig. 1 for 2007-2008 results). Students especially valued the multidisciplinary approach, availability of course content on the Internet, and the small group session with role-playing. They pointed out several weaknesses: excessive content, leading to a too superficial approach; too detailed and specialized content in some sections; some redundancies; too little place for clinical examination and plastic surgery; and for the small group session, too much content and not enough instructors.

\section{9: Perspectives for Future Development}

A framework for the future development of this course can be derived from evaluation results on the one hand and changes in the institutional context on the other hand.

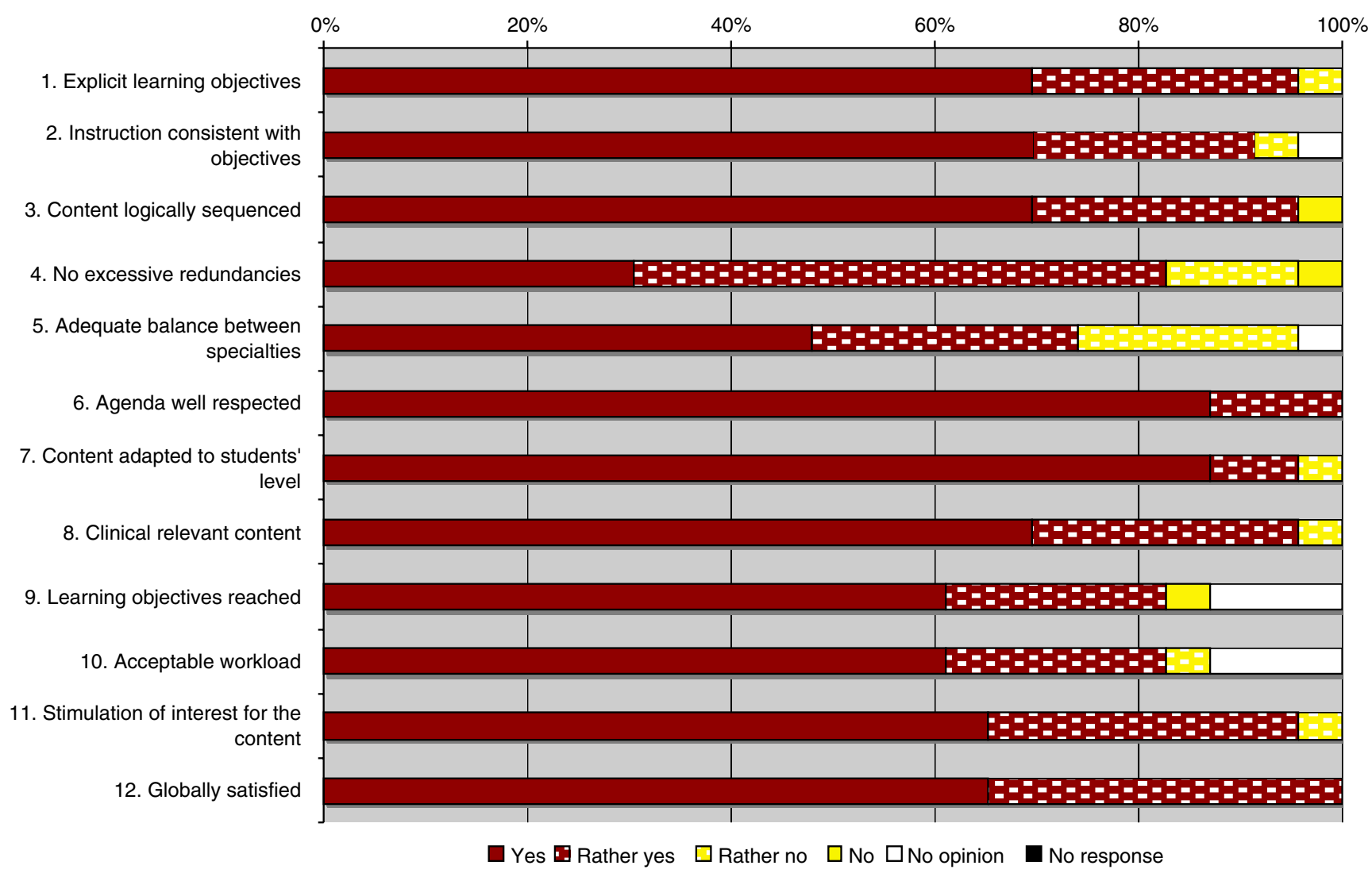

Fig. 1 Results of course evaluation (2007-2008). Twenty-three (23) students, out of around 45 who attended the course, completed a paper questionnaire. Answers were collected and analyzed by the education

unit of the Medical School. The survey also included open-ended questions on strengths and weaknesses of the program (see text) 


\section{Learning Needs and Course Content}

Evaluation results suggest improvements in course content selection, with a larger part to be dedicated to clinical knowledge. The institutional context also indicates ways for improvements, some of which meet students' feedback.

First, the Swiss Catalogue of Learning Objectives, reedited in 2008, classifies breast diseases in the level two category, for which (The doctor is) "able to cope with (the clinical picture) in practice: the physician is able to take a history and perform a physical examination in a structured way. She/he is able to consider the clinical picture as a diagnosis and recognizes and actively searches for the typical complaints and signs. She/he proposes appropriate diagnostic and therapeutic possibilities to her/his supervising physician considering its epidemiology and relevant pathology (including histology, pathophysiology and psychodynamics). She/he explains the diagnostic and therapeutic possibilities to the patient in an appropriate way" [6].Learning objectives, course content, and assessment of knowledge should be further refined in order to match at best these recommendations. This is important for students to be adequately prepared to both their future practice, and the new national final exam, from 2011. It should be noted that the empirical changes in course content are already fairly consistent with these guidelines. A valid assessment of students' learning is however still to be designed.

Second, the experts visiting our Medical School in 1999 as part of a pilot accreditation procedure pointed a need to improve the training of medical students in clinical skills. The new curriculum which started in 2004 introduced a longitudinal course dedicated to training in clinical skills. Breast clinical examination is still to be included in this program while clinical rotations in the gynecology and breast clinic are to be pursued.

\section{Instructional Approach}

The information presented during lectures should be better tailored for undergraduate students. The use of simulation devices for breast examination is under study, as is students' facilitated access to the previously mentioned auto-learning software dedicated to breast clinical examination [5]. Student feedback on the small group session strongly supports the further development of this approach. The material to be worked on should be reduced, and more instructors involved. To model multidisciplinary cooperation, instructors should ideally have experience in breast cancer patient care. Residents and fellows from surgery, oncology, or pathology could fulfill this requirement and learn while participating in instruction.

\section{Discussion}

Multidisciplinary teaching is a major strength of the instructional experience reported in this paper.

Though multidisciplinarity has several theoretical grounds, we view this instructional choice in this context as especially in line with Lave and Wenger's theory of situated learning [7]. Students' learning of principles and facts regarding breast diseases in this program can be expected to be effective because this knowledge is presented by the very physicians who use them in their daily clinical work. The practical session reinforces this strength, by having students actively entering a specialist role. This learning activity allows students to concretely realize physicians' cooperation in breast patients' management.

Multidisciplinary instruction requires coordination, faculty members' good will, and collaboration skills. It harbors at least one threat, the tendency for each specialist to present too much detailed knowledge. Faculty members will have to address this threat in the future, while improving the alignment of the course content with the nationally defined learning objectives for undergraduate students.

Teachers involved in this course are currently involved in the formalization of a multidisciplinary Breast Center. A successful undergraduate-learning module on breast diseases can be regarded as a legitimate purpose for such a Center [8].

An interesting aspect of the experience reported here is how learning and instruction evolved, especially during the past 5 years, as a result of the "co-evolution" of computer technology and educational knowledge.

Digital imaging allowed significant changes in both course content and didactic presentations. Digital supports fit the needs of visual disciplines such as radiology and pathology. This technology also provides students with inexpensive course material, which they can review where and when they want, at their own pace, especially before exams or during a clinical rotation. This progress harbors, however, the threat of "image overload". Selection of images is necessary, in order not to overlook the core learning objectives by presenting too many images illustrating detailed factual information.

The interactive small group session introduced in 2007 links the educational potential of both computer technology and progresses in the understanding of the learning process $[2,4]$. Working actively in small groups, in the context of authentic clinical situations, is expected, according to sociocognitive theories of learning, to motivate students and support knowledge retention and future transfer to practice. Students' enthusiasm about this innovation tends to support the validity of these principles. Discussions within small groups and interactions with instructors allow students to 
discover misunderstandings or partial understandings, and obtain clarification. A partly similar instructional approach, called Team-Based Learning by its proponents, has already been successfully used in cancer education [9].

The main limitation of the experience reported in this paper is that students' knowledge on breast diseases has not been specifically assessed so far, due to the current organization of medical examinations in Switzerland. This will change with the introduction of assessments by semester in the local new curriculum, and of a national comprehensive examination in 2011. The recommendations included in the national catalogue of learning objectives will have to be translated into assessable learning outcomes. The OSCE format will have to be considered to assess examination skills.

\section{In Conclusion}

This paper provides an example of multidisciplinary cancer education. The sustained engagement and cooperation of faculty from several specialties over 15 years resulted in a progressive refinement of learning objectives, course content, and instructional approach. Though the successive changes were rather empirical, Kern's framework for curriculum design allows analyzing past evolutions and outlining future directions. The next challenge for this program will be to adapt to the requirements of a new local whole curriculum, of nationally defined learning objectives, and a new national examination. Caring for this undergraduate program is important as it sets the basis for postgraduate and continuing training of physicians, in the many specialties required for cancer patient efficient care.

This work was supported by a Giorgio Cavaglieri Fund for research in medical education.

\section{References}

1. Choi BC, Pak AW (2006) Multidisciplinarity, interdisciplinarity and transdisciplinarity in health research, services, education and policy: 1. Definitions, objectives, and evidence of effectiveness. Clin Invest Med 29:351-364

2. Mann KV (2001) Thinking about learning: implications for principle-based professional education. J Contin Educ Health Prof 21:90-96

3. Kern DE, Thomas PA, Howard DM, Bass EB (1998) Curriculum development for medical education. A six-step approach. The Johns Hopkins University Press, Baltimore

4. Fiche M, Bonvin R, Bosman F (2006) Microscopes and computers in small-group pathology learning. Med Educ 40:1138-1139

5. Virtual Skills Lab. The gynecological section is only accessible for authorized persons. Available at http://www.virtualskillslab.net. Accessed 7 July 2008

6. Bürgi H, Rindlisbacher B, Bader Ch, et al. (2008) Swiss catalogue of learning objectives for undergraduate medical training. 2nd Edition http://www.smifk.ch

7. Lave J, Wenger E (1991) Situated learning: legitimate peripheral participation. Cambridge University Press, Cambridge

8. EUSOMA (2000) The requirements of a specialist breast unit. Eur J Cancer 36:2288-2293

9. Haidet P, Fecile ML (2006) Team-based learning: a promising strategy to foster active learning in cancer education. J Cancer Education 21:125-128 